\title{
Comprehensive detection of chromosomal translocations in lymphoproliferative disorders by massively parallel sequencing
}

\author{
Philippe Szankasi ${ }^{1}$ (D) Ashini Bolia ${ }^{1}$ - Michael Liew ${ }^{1}$ - Jonathan A. Schumacher ${ }^{1}$ - Elaine P. S. Gee ${ }^{1,2}$. \\ Anna P. Matynia ${ }^{1,3} \cdot$ K. David Li ${ }^{1,3,4} \cdot$ Jay L. Patel ${ }^{1,3} \cdot$ Xinjie Xu ${ }^{1,3} \cdot$ Mohamed E. Salama ${ }^{1,3,5} \cdot$ Todd W. Kelley ${ }^{1,3,6}$
}

Received: 25 April 2019 / Accepted: 17 June 2019/Published online: 1 July 2019

(C) Springer-Verlag GmbH Germany, part of Springer Nature 2019

\begin{abstract}
Balanced translocations have diagnostic and prognostic value in B-cell lymphoproliferative disorders (LPDs). Most of these translocations involve the juxtaposition of a strong immunoglobulin (Ig) enhancer to proto-oncogenes, such as BCL2, BCL6, and $M Y C$, leading to their overexpression. These rearrangements generally do not result in mRNA fusions, and fluorescent in situ hybridization (FISH) remains the gold standard for assessing of recurrent translocations in LPDs. With the growing use of massively parallel sequencing for the detection of both point mutations and large structural rearrangements, we aimed at evaluating the utility of this method for the molecular work-up of B-cell LPDs side by side with FISH. We describe a method using solution capture for enrichment of known translocation breakpoints and massively parallel sequencing for the detection of balanced translocation in formalin-fixed tissues with a B-cell LPD. We detected a total of 57 rearrangements with a high concordance of $94.2 \%$ when compared to FISH. We detected translocations between BCL2, BCL6, and MYC and the three Ig loci and non-Ig loci, including novel partners for MYC and BCL6. In addition, massively parallel sequencing allowed a detailed analysis of the structure of the resulting chromosomal fusions. Our comparison shows the feasibility of using massively parallel sequencing for detecting balanced translocations in B-cell LPDs and advantages and disadvantages to both methods, and how they can complement each other.
\end{abstract}

Keywords Chromosomal translocation $\cdot$ Massively parallel sequencing $\cdot$ B-cell lymphoma $\cdot$ VDJ recombination

\section{Introduction}

B-cell lymphoproliferative disorders (LPDs) often demonstrate characteristic translocations involving one of the 3

Electronic supplementary material The online version of this article (https://doi.org/10.1007/s12308-019-00360-0) contains supplementary material, which is available to authorized users.

Philippe Szankasi

philippe.szankasi@aruplab.com

1 ARUP Laboratories, 500 Chipeta Way, Salt Lake City, UT 84108, USA

2 Present address: BigHead Analytics Group, Windsor, CA, USA

3 Department of Pathology, University of Utah, Salt Lake City, UT, USA

4 Present address: Department of Pathology, University of Washington, Seattle, WA, USA

5 Present address: Mayo Clinic, Rochester, MN, USA

6 Present address: Navican, Salt Lake City, UT, USA immunoglobulin (Ig) loci. This is due to aberrant processing of programmed DNA breaks introduced during $\mathrm{V}$ (D) J recombination, class switch recombination, or AID-mediated somatic hyper mutation (SHM) in the three immunoglobulin loci $[1,2]$. The hallmark of these translocations is that they do not result in the classical mRNA and protein fusions but instead juxtapose strong enhancer elements of the Ig loci to proto-oncogenes, such as BCL2, BCL6, CCND1, MYC, and MALT1, leading to their overexpression. These types of translocations can therefore only be detected by analyzing genomic DNA and not cDNA. The wide distribution of breakpoints, especially in the partner genes, results in poorer detection rates of these events by PCR and routine testing for the common $t(14 ; 18)$; $I G H-B C L 2$, for example, by PCR is typically focused on those events involving the most common breakpoint cluster regions which markedly limits the sensitivity of the method [3].

Massively parallel sequencing has been used successfully not only for the detection of point mutations and small insertions and deletions but also for detecting structural rearrangements and copy number variants [4]. Because of the growing number of actionable gene mutations and the potential need to 
assess multiple translocations to identify so-called double-hit lymphomas, massively parallel sequencing may provide an economic single-platform tool for the molecular work-up of B-cell LPDs and other cancers. Although most of the breakpoints in the Ig loci occur at defined locations, those in the partner genes can be distributed over large areas of up to $100 \mathrm{~s}$ of $\mathrm{kb}$, making it nearly impossible to fully sequence all regions involved. In addition, for some genes, such as BCL6, many potential fusion partners have been observed $[5,6]$. Solution capture is a powerful method for the enrichment of genomic targets for massively parallel sequencing and, in the case of translocations and other rearrangements, allows for the identification of unknown and untargeted fusion partners/ regions by virtue of their proximity to known, captured sequences in fusion fragments [7]. Here, we evaluate a method based on solution capture enrichment and massively parallel sequencing for the comprehensive detection of translocations in B-cell LPDs in comparison to FISH.

\section{Materials and methods}

\section{Patient specimens}

The use of leftover specimens was approved by the University of Utah Institutional Review Board (IRB\# 78271).

\section{Genomic DNA isolation and sequencing}

DNA was prepared from four 5- $\mu \mathrm{m}$ FFPE tissue scrolls, fragmented to approximately $150 \mathrm{bp}$ by sonication on the Covaris LE220 instrument (Covaris, Inc., Woburn, MA), and massively parallel sequencing libraries were prepared using the Kapa Hyper Prep Kit (Kapa Biosystems, Inc., Wilmington, MA). Illumina TruSeq adapters with an 8-base sample index and a $16 \mathrm{~N}$-unique molecular identifier (UMI) were used for sequencing library preparation. Target enrichment using SureSelect capture (Agilent Technologies, Santa Clara, CA) was performed according to the manufacturer's protocol. Libraries were sequenced on an Illumina NextSeq or MiSeq instrument using $2 \times 150$-base paired-end sequencing (Illumina, Inc., San Diego, CA).

\section{Capture probe design}

Areas of 400 base pairs (bp) surrounding each recombination signal sequence (RSS) of the $V, D$, and $J$ segments of the $I G H$, $I G K$, and $I G L$ loci, approximately 5-6 kilobasepairs $(\mathrm{kb})$ harboring each IGH switch region, and 600 and 1500 bp harboring the intronic and downstream kappa deleting elements, respectively, were selected. Due to close proximity, the $J_{K}$ segments were captured as a single large region and one 10$\mathrm{kb}$ region spanning from $D_{H} 7-27$ to the switch $\mu$ region, including the $J_{H}$ elements and the $\mu$ enhancer, was captured. Genomic regions harboring known breakpoint clusters at the Ig translocation partner genes BCL2, BCL6, MYC, CCND1, $M A L T 1$, and BIRC3 were also captured based on published coordinates [5, 8-14]. See Supplemental Table S1 for a list of all captured regions.

\section{Sequencing data analysis}

Paired-end FASTQ reads were aligned to the human genome reference sequence (b37) using BWA mem [15] with supplemental (chimeric) reads retained, and PCR duplicates were eliminated based on the $16 \mathrm{~N}$ UMI sequence using an inhouse PCR de-duping with UMI tool, BMFTools (https:// github.com/ARUP-NGS/BMFtools v1.0.2). Two types of alignments were performed in the translocation variant calling pipeline, where standard full-length read alignments were supplemented with a second alignment of reads trimmed back to the first 30 bases to facilitate discordant paired-end read support. These two resulting BAM files were merged, and intra- and inter-chromosomal structural variants were called from this merged BAM by DELLY v.0.7.2 [16], which utilizes read pairs that map to different chromosomes/loci (discordant read pairs) and "supplemental" reads with chimeric alignment (split reads) to detect translocations. Translocation calls require a minimum mapping quality of 30 and at least 5 supporting discordant read pairs for inter-chromosomal translocations and 3 for intra-chromosomal events, such as deletions, duplications, and inversions. The identified genomic breakpoint coordinates were annotated with the name of the region or gene in which they overlap with exon-level resolution by a custom python script using information from the UCSC Table Browser (https:/genome.uscs.edu/cgi-bin/ hgTables).

\section{FISH analysis}

FISH analysis was carried out essentially as described [17] using Vysis LSI IGH/BCL2 Dual Color Fusion Probe, Vysis LSI BCL6 (ABR) Dual Color Break Apart Probe, or Vysis LSI MYC Dual Color Break Apart Probe, and for cases 22, 31, and 32 only (see text), Vysis LSI IGH Dual Color Break Apart Probe (Abbott, Abbott Park, IL). At least 100 cells were scored.

\section{Cell culture}

The cell lines Raji, Ramos, and OCI-LY1 were obtained from ATCC (Manassas, VA), and the cell lines REC and NCEB were generously provided by Dr. Elenitoba-Johnson (University of Pennsylvania, Dept. of Pathology). The cell lines SUDHL-4, SUDHL-1, and SUDHL-16 were from a collection at ARUP. All cell lines were grown in RPMI1640 
supplemented with $10 \%$ fetal bovine serum at $5 \% \mathrm{CO}_{2}$. In dilution experiments, cells were counted with a hemocytometer and pellets of 50-100 million cells were formalin fixed and paraffin embedded.

\section{Results}

\section{Detection of translocations by massively parallel sequencing}

We tested 66 patient specimens, most of which had been given a diagnosis of B-cell LPD, including follicular lymphoma (FL; $n=24$ ), diffuse large B-cell lymphoma (DLBCL; $n=$ $21)$, cases with both FL and DLBCL $(n=2)$, Burkitt lymphoma (BL; $n=4)$, and extranodal marginal zone B-cell lymphoma of MALT-type (MALT lymphoma; $n=9$ ). We also tested a fresh bone marrow core sample from a patient with B-ALL harboring a known $I G H-C R L F 2$ fusion. For a list of patient characteristics, see Table 1 . In addition, 7 established reference B-cell lines were processed. Table 2 lists the detected translocations annotated with the breakpoint regions along with the confirmatory FISH results. Out of the 66 patient samples tested, 50 samples harbored a total of 57 rearrangements identified by sequencing with 7 samples harboring 2 rearrangements, each. We detected 30 translocations between $I G H$ and BCL2, 8 between BCL6 and IGH, 6 between MYC and $I G H, 3$ between BIRC3 and MALT1, and one each between the 2 light chain loci and BCL6 and MYC. We further detected translocations between BCL6 and 4 non-Ig partners, NACA, CIITA, IL21R, and DLEU2, 3 of which have previously been described $[5,18,19]$. Case 22 harbored a fusion between $M Y C$ and a non-Ig partner, $S T X 7$. No RNA fusion is predicted between $M Y C$ and $S T X 7$ since the 2 genes are transcribed in opposite directions but the region fused to the $M Y C$ promotor, $S T X 7$ intron 1, contains an area of strong histone $\mathrm{H} 3$ Lys 27 acetylation (based on ENCODE data viewed in the UC Santa Cruz Genome Browser, www.genome.ucsc.edu, last accessed June 29, 2016, Supplemental Fig. S1), consistent with transcriptional enhancer activity. An $I G H-C R L F 2$ fusion was detected in case 66 .

The output by the DELLY software does not estimate a variant allele frequency (VAF) for structural rearrangements but does report various read counts, such as junction reads. To determine a limit of detection for translocations by this method, we diluted cell lines with known translocations (Table 2) into a cell line negative for the tested translocations. Equal cell numbers of the six cell lines SUDHL-4, SUDHL-16, OCILY1, RAJI, REC, and NCEB were mixed and further diluted into the negative cell line SUDHL- 1 to a final concentration of $20 \%, 10 \%$, and $5 \%$ each, respectively. All six expected translocations could be detected down to $5 \%$ positive cells but reproducible detection of both derivative chromosomes (see also below) was only possible down to $10 \%$ positive cells. In the mixed sample with $10 \%$ each of the six cell lines, DELLY correctly called all 12 resulting reciprocal fusion products (derivative chromosomes) even though some of the breakpoints mapped to sequences in close proximity (within $1 \mathrm{~kb}$ ).

\section{Concordance between sequencing and FISH}

Six cases (cases 15-20) were selected for this analysis because of a prior positive FISH result. For the remaining cases with a translocation detected by sequencing (excluding BIRC3-MALT1) and 9 cases negative by sequencing, confirmatory FISH analysis was performed using fusion probes for $I G H-B C L 2$ and break-apart probes for BCL6 and $M Y C$. See Table 2 for all FISH results. Three additional cases $(63,64$, and 65$)$ were sequenced because an atypical FISH pattern was observed that precluded interpretation of the FISH results. Out of 53 translocations, initially detected by either FISH or sequencing, four yielded discordant results (Table 2). In case 9, a breakpoint identified by sequencing mapped $835.5 \mathrm{~kb}$ upstream of $B C L 6$, in the $L P P$ gene. This position is outside the region covered by the break-apart BCL6 FISH probe used and was therefore not detected. Case 11 yielded no translocations by sequencing but was weakly positive by $M Y C$ break-apart FISH (24\% positive nuclei). Case 17, which demonstrated focal positivity by MYC break-apart FISH ( $80 \%$ positive nuclei in this region), was also negative by sequencing. However, it is important to note that no enrichment of tumor tissue was performed in this case or on any of the specimens tested by sequencing. An $I G H-B C L 2$ translocation detected by FISH in case 46 was not called by DELLY but reciprocal fusion sequences at the BCL2-icr cluster region could be identified manually using IGV software. The corresponding discordant read pairs aligned poorly to the $I G H$ locus, possibly due to high focal homologies between the RSS sites of multiple $V_{H} 2$ family members (data not shown). Figure 1 summarizes the concordance between sequencing and FISH. The three translocations that yielded atypical staining patterns by FISH and were positive for 2 out of the 3 putative translocations by sequencing (cases 63-65, Table 2) were not included in Fig. 1. In summary, 49/52 (94.2\%) translocations detected by FISH could be confirmed by sequencing and 49/50 (98\%) translocation detected by sequencing were confirmed by FISH. Out of 24 FL cases, one $(4.2 \%)$, and out of 15 DLBCL cases, three (20\%) yielded discordant results between sequencing and FISH.

\section{Fusion sequence analysis}

Identification of translocations and other structural rearrangements by massively parallel sequencing allows determination 
Table 1 Patient characteristics

\begin{tabular}{|c|c|c|c|c|}
\hline Case & Diagnosis $^{\mathrm{a}}$ & Specimen $^{\mathrm{b}}$ & Sex & Age \\
\hline 1 & DLBCL, NOS & $\mathrm{LN}$ & M & 67 \\
\hline 2 & DLBCL, NOS & Parotid mass & M & 73 \\
\hline 3 & DLBCL, NOS & $\mathrm{LN}$ & $\mathrm{F}$ & 24 \\
\hline 4 & DLBCL, NOS & $\mathrm{LN}$ & M & 62 \\
\hline 5 & DLBCL & $\mathrm{LN}$ & $\mathrm{F}$ & 68 \\
\hline 6 & DLBCL, NOS & Left neck mass & M & 85 \\
\hline 7 & DLBCL, GCB & Left groin mass & M & 69 \\
\hline 8 & DLBCL & $\mathrm{LN}$ & M & 70 \\
\hline 9 & DLBCL & $\mathrm{LN}$ & $\mathrm{F}$ & 80 \\
\hline 10 & DLBCL, NOS & $\mathrm{LN}$ & $\mathrm{F}$ & 62 \\
\hline 11 & DLBCL, NOS & Thyroid isthmus & $\mathrm{F}$ & 89 \\
\hline 12 & DLBCL, NOS & Ovary, salpingo & $\mathrm{F}$ & 40 \\
\hline 13 & DLBCL & $\mathrm{LN}$ & M & 85 \\
\hline 14 & DLBCL, NOS & Left parascapular & M & 64 \\
\hline 15 & DLBCL & $\mathrm{LN}$ & M & 42 \\
\hline 16 & DLBCL, ABC & Right neck mass & M & 66 \\
\hline 17 & DLBCL & Cardiac mass & M & 57 \\
\hline 18 & Hg DLBCL, GCB & $\mathrm{LN}$ & M & 30 \\
\hline 19 & DLBCL, NOS, ABC & $\mathrm{LN}$ & $\mathrm{F}$ & 60 \\
\hline 20 & DLBCL, non-GCB & $\mathrm{LN}$ & M & 79 \\
\hline 21 & FL, grade 3 , DLBCL & Bowel and mesentery & M & 81 \\
\hline 22 & FL, grade 3, DLBCL & $\mathrm{LN}$ & M & 59 \\
\hline 23 & DLBCL from CLL & $\mathrm{LN}$ & $\mathrm{F}$ & 60 \\
\hline 24 & CLL/DLBCL, FL, grade 2-3 & $\mathrm{LN}$ & $\mathrm{F}$ & 74 \\
\hline 25 & $\mathrm{Hg} \mathrm{BCL}$ & Left submandibular mass & M & 36 \\
\hline 26 & FL, grade $1-2$ & $\mathrm{LN}$ & M & 55 \\
\hline 27 & FL, grade $1-2$ & $\mathrm{LN}$ & M & 62 \\
\hline 28 & FL, grade $1-2$ & $\mathrm{LN}$ & M & 90 \\
\hline 29 & FL & LN, needle core & $\mathrm{F}$ & 77 \\
\hline 30 & FL, grade $1-2$ & Soft tissue, elbow & $\mathrm{F}$ & 26 \\
\hline 31 & FL, low grade & Axilla, needle core & $\mathrm{F}$ & 59 \\
\hline 32 & FL, grade $1-2$ & $\mathrm{LN}$ & $\mathrm{F}$ & 78 \\
\hline 33 & FL, grade 2 & Sigmoid colon & $\mathrm{F}$ & 83 \\
\hline 34 & FL, grade 1 & Retroperitoneal & M & 67 \\
\hline 35 & FL, grade $2-3$ & $\mathrm{LN}$ & $\mathrm{F}$ & 58 \\
\hline 36 & FL, grade 3 & $\mathrm{LN}$ & $\mathrm{F}$ & 58 \\
\hline 37 & FL, grade 1 & Epitrochlear LN & M & 55 \\
\hline 38 & FL, grade $1-3$ & Left parotid mass & $\mathrm{F}$ & 71 \\
\hline 39 & FL, grade $1-2$ & $\mathrm{LN}$ & M & 84 \\
\hline 40 & FL, grade 2 & Left lower lid & $\mathrm{F}$ & 67 \\
\hline 41 & FL, grade $1-2$ & $\mathrm{LN}$ & $\mathrm{F}$ & 57 \\
\hline 42 & FL, grade 2 & Duodenal nodule & M & 66 \\
\hline 43 & FL, grade 3 & Abdominal mass, needle core & $\mathrm{F}$ & 75 \\
\hline 44 & FL, grade $1-3$ & $\mathrm{LN}$ & $\mathrm{F}$ & 70 \\
\hline 45 & FL, grade $1-3$ & $\mathrm{LN}$ & M & 80 \\
\hline 46 & FL, grade $2-3$ & $\mathrm{LN}$ & $\mathrm{F}$ & 90 \\
\hline 47 & FL, grade $1-2$ & $\mathrm{LN}$ & $\mathrm{F}$ & 89 \\
\hline 48 & FL, grade $1-2$ & Left groin mass & M & 76 \\
\hline 49 & $\mathrm{FL}$, grade $2-3$ & $\mathrm{LN}$ & M & 56 \\
\hline
\end{tabular}


Table 1 (continued)

\begin{tabular}{lllll}
\hline Case & Diagnosis $^{\mathrm{a}}$ & Specimen $^{\mathrm{b}}$ & Sex & Age \\
\hline 50 & $\mathrm{BL}$ & $\mathrm{LN}$ & $\mathrm{M}$ & 56 \\
51 & $\mathrm{BL}$ & Liver & $\mathrm{F}$ & 49 \\
52 & $\mathrm{BL}$ & Ileum, cecum, appendix & $\mathrm{M}$ & 32 \\
53 & $\mathrm{BL}$ & Tongue & $\mathrm{M}$ & 21 \\
54 & MALT lymphoma & Right period mass & $\mathrm{F}$ & 42 \\
55 & MALT lymphoma & Lung, right lobes & $\mathrm{M}$ & 62 \\
56 & MALT lymphoma & Stomach & $\mathrm{M}$ & 56 \\
57 & MALT lymphoma & Stomach & $\mathrm{F}$ & 67 \\
58 & MALT lymphoma & Cecal polyp & $\mathrm{M}$ & 72 \\
59 & MALT lymphoma & Stomach & $\mathrm{F}$ & 81 \\
60 & MALT lymphoma & Small bowel mass & $\mathrm{F}$ & 65 \\
61 & MALT lymphoma & Left parotid gland & $\mathrm{M}$ & 59 \\
62 & MALT lymphoma & Lung, right middle lobe & $\mathrm{M}$ & 52 \\
63 & Unknown & Gastric mass & $\mathrm{M}$ & 57 \\
64 & Unknown & Left chest wall & $\mathrm{M}$ & 71 \\
65 & Unknown & Right neck mass & M & 29 \\
66 & B-ALL & BM core & & 56 \\
\hline
\end{tabular}

${ }^{\text {a }} D L B C L$, diffuse large B-cell lymphoma; $N O S$, not otherwise specified; $G C B$, germinal center B-cell type; $A B C$, activated B-cell type; $H g$, high-grade; $F L$, follicular lymphoma; $C L L$, chronic lymphocytic leukemia; $B C L$, B-cell lymphoma; $B L$, Burkitt lymphoma; $M A L T$, mucosa-associated lymphoid tissue; $B-A L L$, B-cell lymphoblastic leukemia

of the precise structure and identity of the fused chromosomal regions. We used the Integrated Genome Viewer (IGV) program to visualize the nature of the detected fusions at the single nucleotide level, based on split reads that span translocation breakpoints. Figure 2 shows images from the IGV program for the two translocations in case 36 . Uniformly colored bars indicate reads aligned to the reference genome. Bases listed in the reads indicate mismatches. Split reads either align left and are mismatched on the right, or vice versa, providing a visual representation of reads derived from the two derivative chromosomes (Fig. 2a).

\section{Distribution of $I G H-B C L 2$ breakpoints}

As has previously been described [20-22], we detected two $I G H$ breakpoints for a majority $(27 / 33,81.8 \%)$ of $I G H-B C L 2$ translocations, one in a $J$ segment and the other in a $D$ segment, consistent with aberrant $D-J$ recombination [Table 2, most $\mathrm{t}(14 ; 18)$ cases]. For example, in case 36 (Fig. 2b), reads from only one derivative breakpoint are aligned to the $J_{H} 6$ segment and reads from the other derivative are aligned to the $D_{H} 2-2$ segment, indicating a deletion of the intervening $53 \mathrm{~kb}$ from the translocated chromosome 14 . In cases 31 and 38, the BCL2 sequences were fused to $I G H$ in an inverted orientation with respect to a classical $t(14 ; 18)$. In these cases, a second fusion point at the other end of the $B C L 2$ locus was detected, indicating an insertion of the entire BCL2 locus into $I G H$ (between
$J_{H} 3$ and $D_{H} 3-22$ or at $J_{H} 5$, respectively, Fig. 3a). These rare events are attributed to a possible transposase-like function of the Rag proteins [23]. The nature of this insertion in case 31 was corroborated by a negative FISH result with $I G H$ breakapart probes. These probes map to either side of the site of BCL2 insertion (Fig. 3a) and are not expected to be separated by the rearrangement. Two control cases with a classical $\mathrm{t}(14 ; 18)$, cases 22 and 32, were positive by $I G H$ break-apart FISH (data not shown). The 2 cases with BCL2 inserted into $I G H$ were still positive by FISH with $I G H-B C L 2$ fusion probes which span the entire loci (Fig. 3a). For the 30 BCL2 rearrangements detected in patient samples, Fig. 4 shows the distribution of the 32 breakpoints detected in BCL2. As previously established, most of these breakpoints occurred in the major breakpoint cluster region (MBR). Only 3/35 observed breakpoints were not captured. Standard PCR-based tests for $I G H-B C L 2$ typically use primers in the MBR and mor regions and would potentially detect $21 / 30(70 \%)$ of the events observed by sequencing.

\section{Non-Ig fusions of $B C L 6$}

Figure $3 \mathrm{~b}$ shows the fusion gene structure between BCL6 and the non-Ig partners CIITA, NACA, and DLEU2, based on the split read sequences (for example Fig. 2a for case 36), likely bringing BCL6 under the control of a heterologous promotor. These BCL6 fusions to non-Ig partners generally result in 5'- 


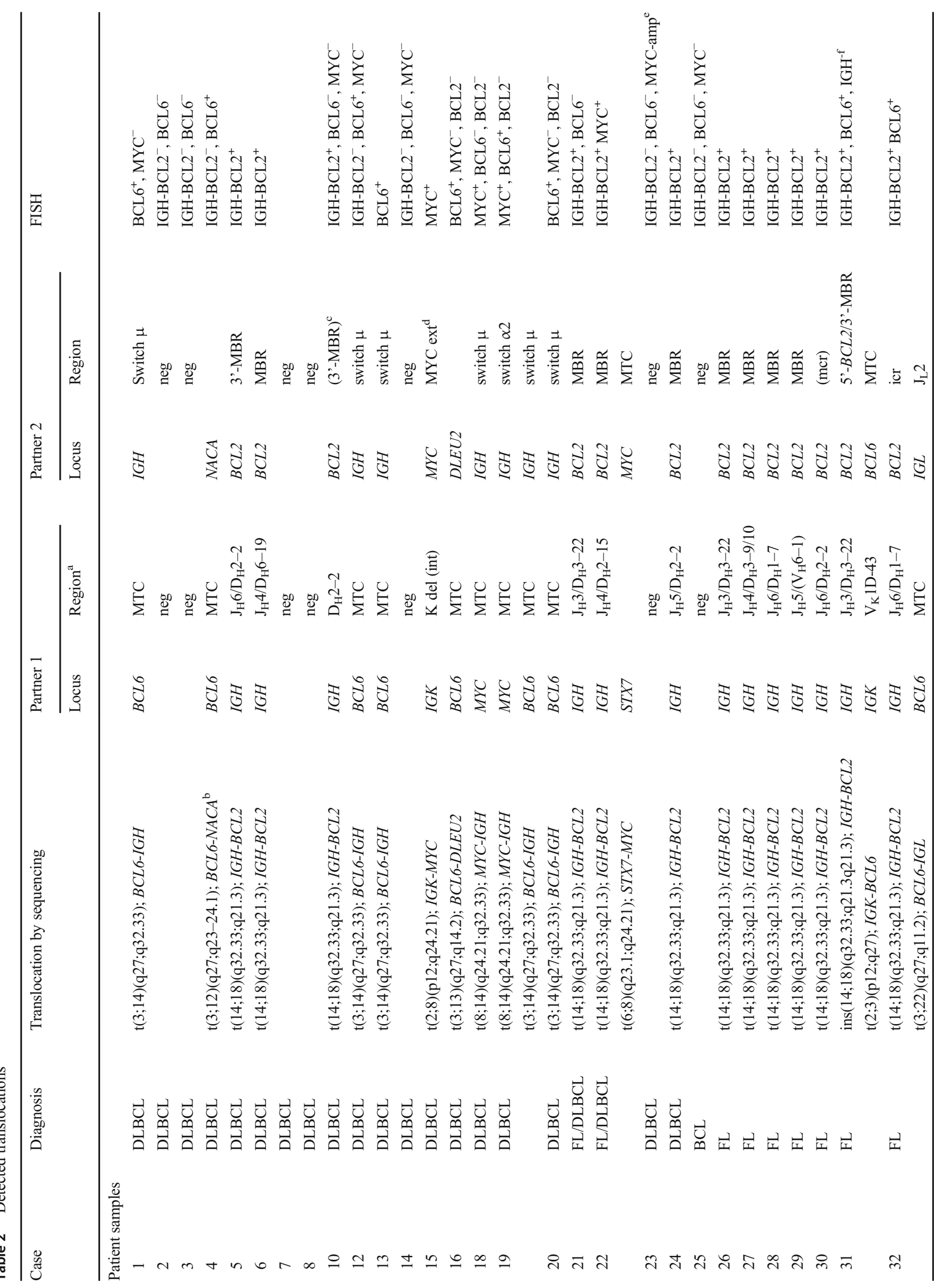




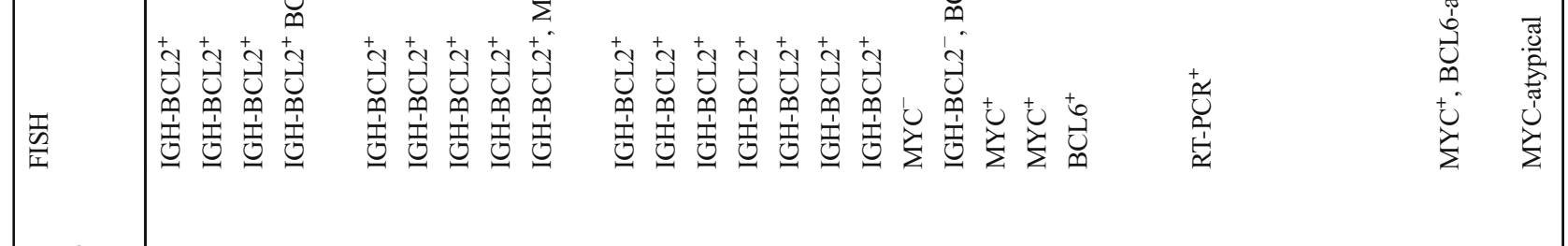

$\overbrace{0}^{+\infty}$

$\stackrel{t}{ \pm}$

$\stackrel{ \pm}{Z}$

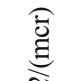

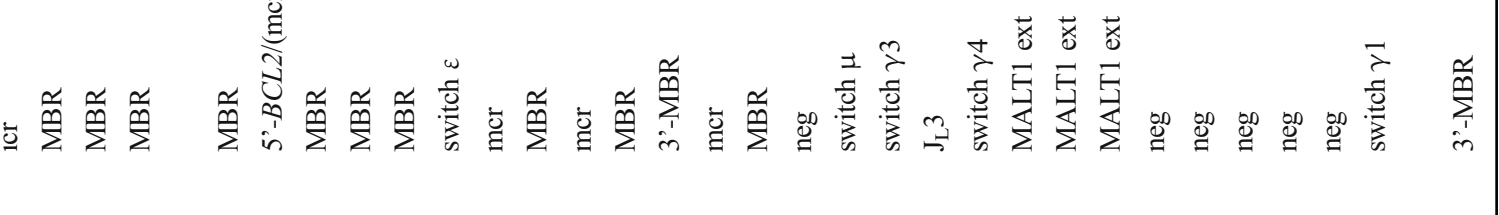
芩苍论

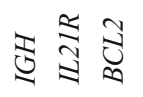

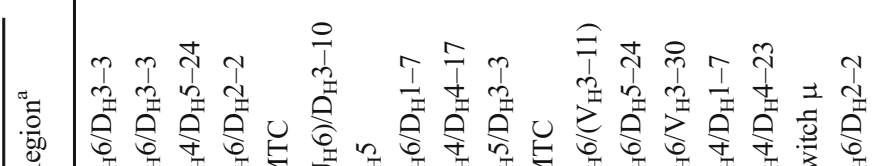

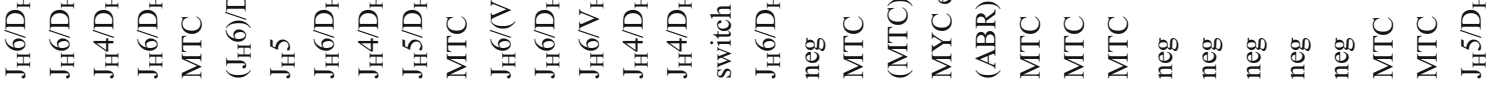

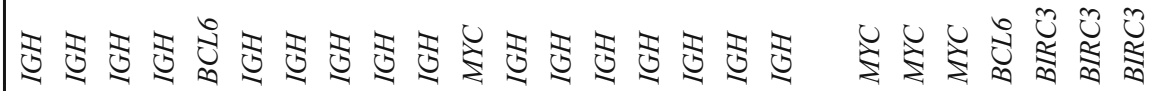

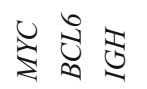

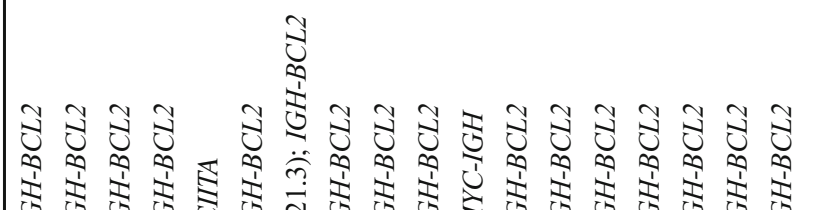

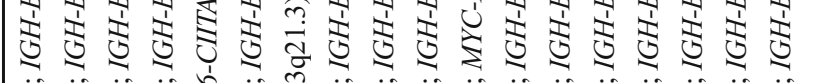

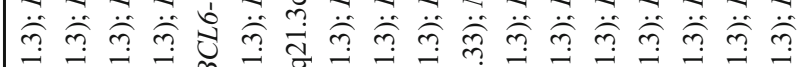

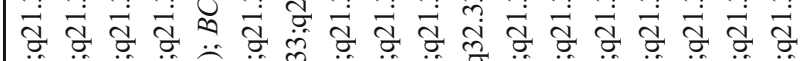

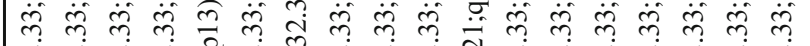

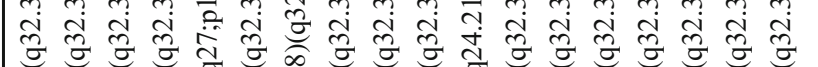

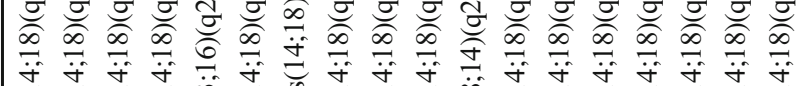

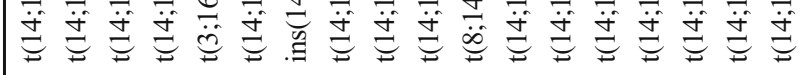

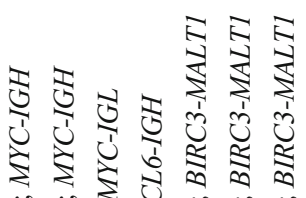

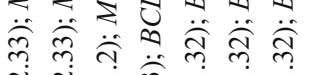
ते

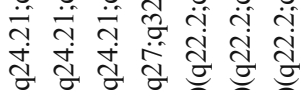

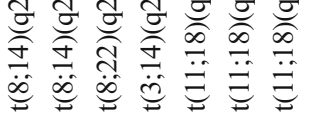

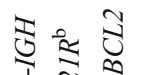

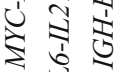
药 (

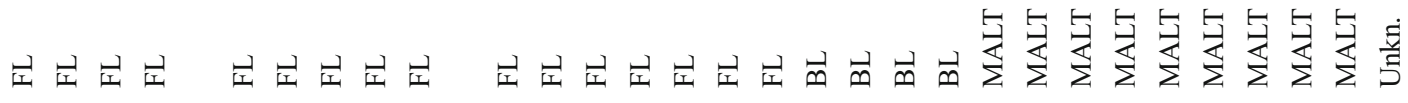
तิ

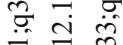

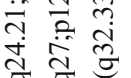
过

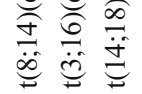

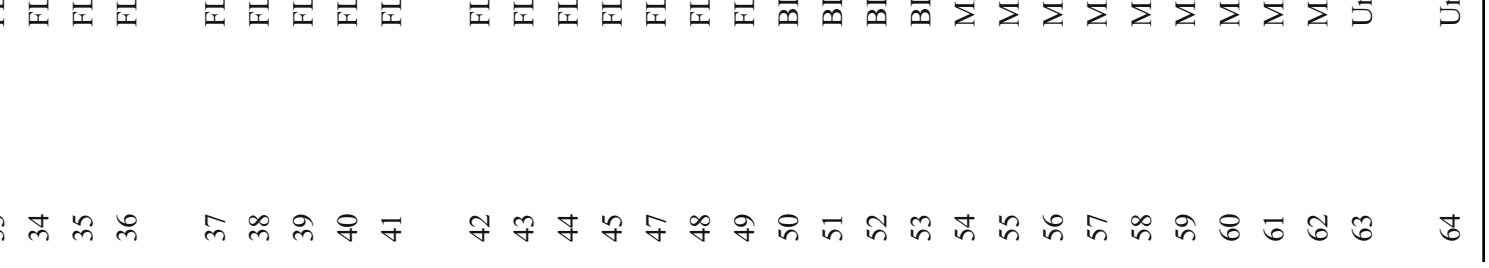




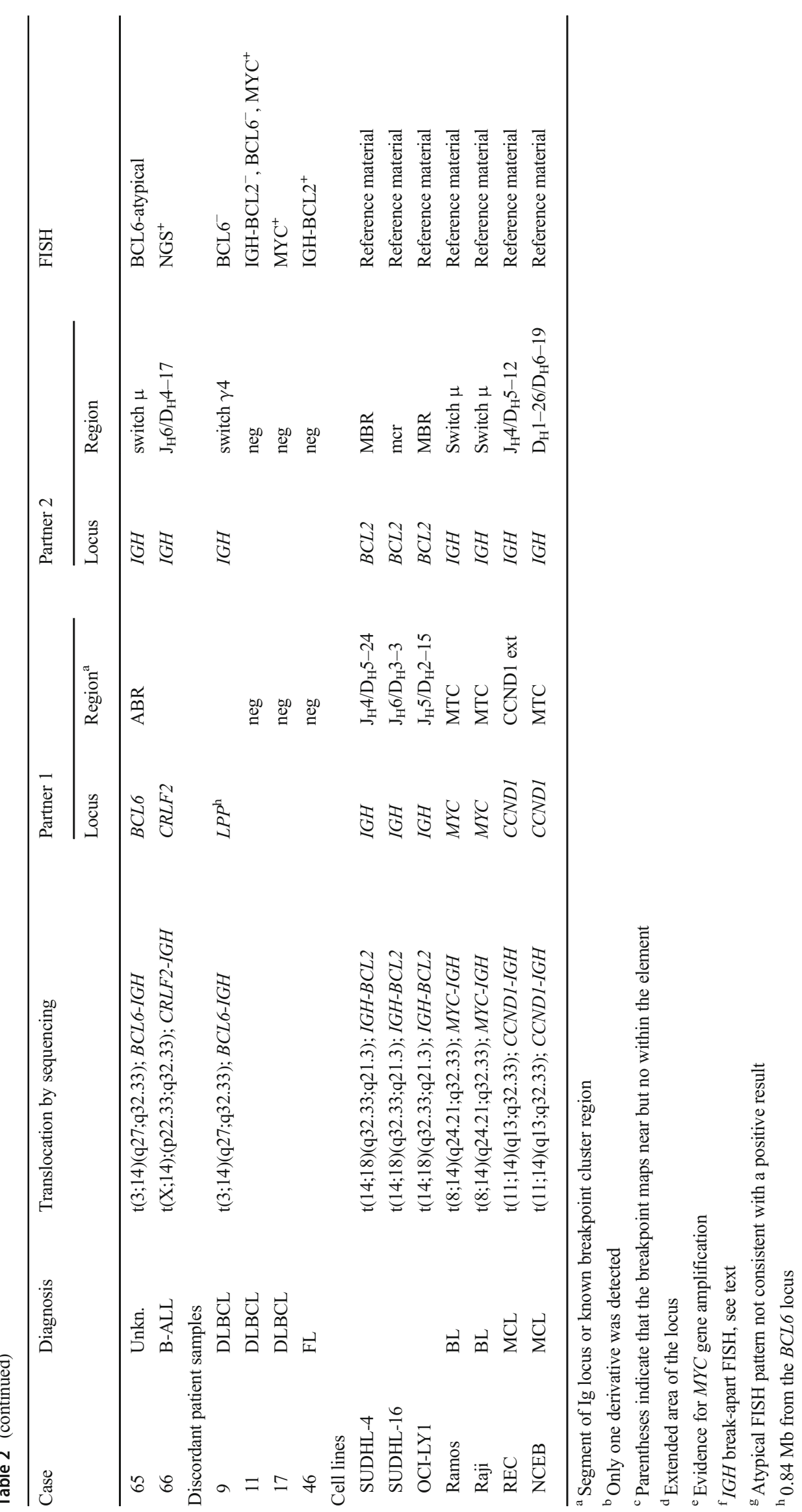




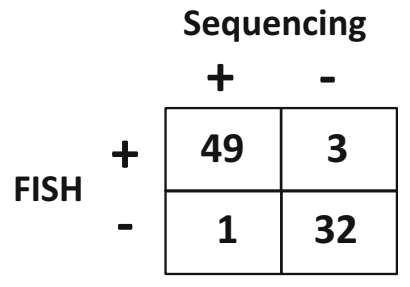

Fig. 1 Concordance between massively parallel sequencing and FISH. Individual translocations in patient samples with both sequencing and FISH data are listed. Negative sequencing and FISH data are also listed

UTR fusions [5, 6] and should therefore also be detectable by RNA sequencing methods. The only other class of mRNA fusions observed in this study are represented by the 3 MALT lymphoma cases $(55-57)$ with a t(11;18); BIRC3MALT1 translocation.

\section{Distribution of Ig breakpoints}

$I G H$ breakpoints were detected in many of the possible elements involved in $D-J$ recombination $\left(13 / 27 D_{H}\right.$ elements, 4/6 $J_{H}$ elements) and in 6/8 class switch regions. As has previously been described, $I G H$ was the most common Ig locus involved in translocations. Among the 57 translocations identified in 50 cases, 45 (79\%) involved $I G H, 2$ involved $I G K$, and 2 involved $I G L$. Translocation breakpoints were detected in all previously described cluster regions of the non-Ig genes, validating the comprehensive capture design.

\section{Detection of balanced rearrangements}

For 53/57 translocations (93\%), the fusion fragments from both derivative chromosomes could be detected, corroborating the balanced structure of these rearrangements. Of the remaining 4 cases with only one derivative chromosome detected, $2(31,38)$ represent insertions of the BCL2 locus into $I G H$ that may not be balanced (see above). The additional 2 cases likely harbor associated deletions or a complex event. When only one translocation partner is captured, a breakpoint that is associated with a deletion extending beyond the captured area leads to the inability to capture both derivative fusion fragments. For example, in case 4, only those split reads supporting the $B C L 6-N A C A$ fusion diagramed in Fig. 3 but not the reciprocal product were observed, whereas BCL6 break-apart FISH analysis was unequivocal for a BCL6 translocation (signal pattern = 1 fused, 1 red, 1 green; data not shown), indicating the presence of both derivatives at the megabase level. Case 63 shows the previously reported rearrangement between the $B C L 6$ and $I L 21 R$ loci [18] but only the presumed non-oncogenic fusion product was detected. The BCL6 FISH pattern was atypical (data not shown) and this case may have a complex structure not discernible by targeted sequencing of genomic DNA.

\section{Capture of unknown fusion sequences}

Out of the 64 detected translocations (in 50 patient samples and 7 cell lines), 17 (26.5\%) were identified by capturing only one of the fusion partners. These include the 5 translocations involving the non-Ig partners of BCL6 and $M Y C$ and translocations with breakpoints mapping $100 \mathrm{~s}$ of kb from known target genes, such as BCL6, MYC, or $C C N D 1$, for example cases 9 and 53 and the cell line REC. Multiple breakpoints were also identified just outside $(0.5$ to $6 \mathrm{~kb})$ of well-defined cluster regions (for example, cases 10, 30, 52, 54).

We detected only two translocations where an Ig breakpoint could not be captured, occurring $4.3 \mathrm{~kb}$ downstream of $V_{H} 6-1$ (case 29) and $21 \mathrm{~kb}$ downstream of $V_{H} 3-11$ (case 42), respectively.

\section{Discussion}

Here, we describe a method using massively parallel sequencing for the detection of translocations in B-cell lymphoproliferative disorders. Our comprehensive capture of the sites of programmed DNA breaks in the $3 \mathrm{Ig}$ loci and the well-characterized breakpoint cluster regions in the known partner genes lead to the successful identification of multiple translocations between the Ig loci and the proto-oncogenes BCL2, BCL6, MYC, and CCND1. In addition, we detected non-Ig fusions for BCL6 and $M Y C$, and translocations between MALT1 and BIRC3. Many of the captured elements involved in regular $V(D) J$ recombination, class switch recombination, and kappa deletion were found to be involved in the translocation events. We observed a high concordance with confirmatory FISH analysis (Fig. 1), with $94.2 \%$ of FISH-positive results confirmed by sequencing.

The 3 FISH-positive cases that could not be confirmed by massively parallel sequencing may illustrate some of the challenges of this method and the potential contributing factors should be considered. Although the cell line dilution experiment presented here showed detection of translocations by sequencing in a sample with as few as $10 \%$ positive cells, some clinical samples may contain a significant portion of normal tissue, which serves to dilute the abnormal reads derived from translocations. No microdissection of the tissues was carried out in this study and this may mean that these cases simply had too few positive cells for translocation detection by this method (see Supplemental Fig. S2 for H\&E slide showing patchy distribution of tumor for case 17). 


\section{a $\mathrm{t}(3 ; 16)(q 27 ; p 13) ; B C L 6-C I I T A$}

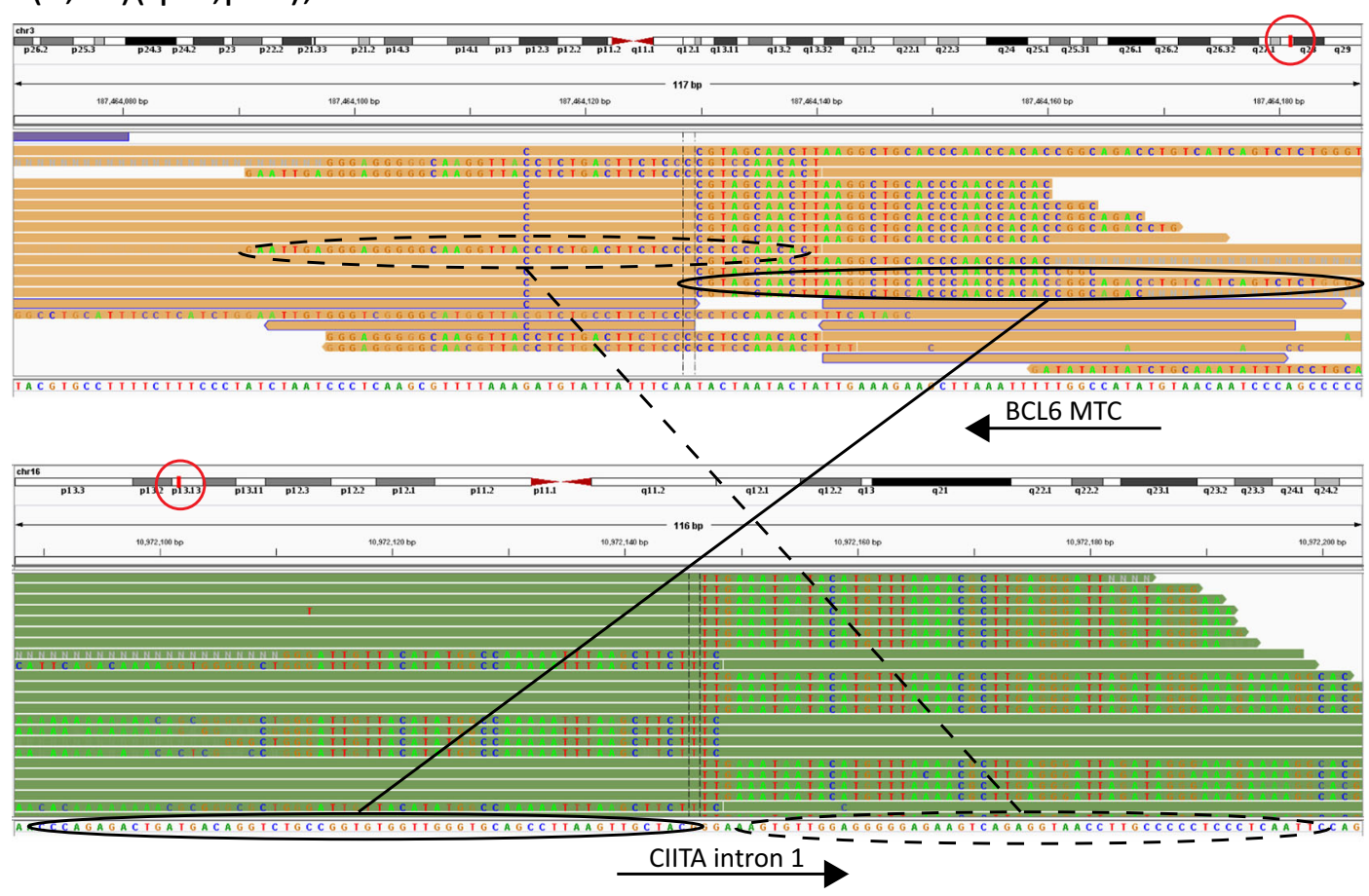

b t(14;18)(q32.33;q21.3); IGH-BCL2

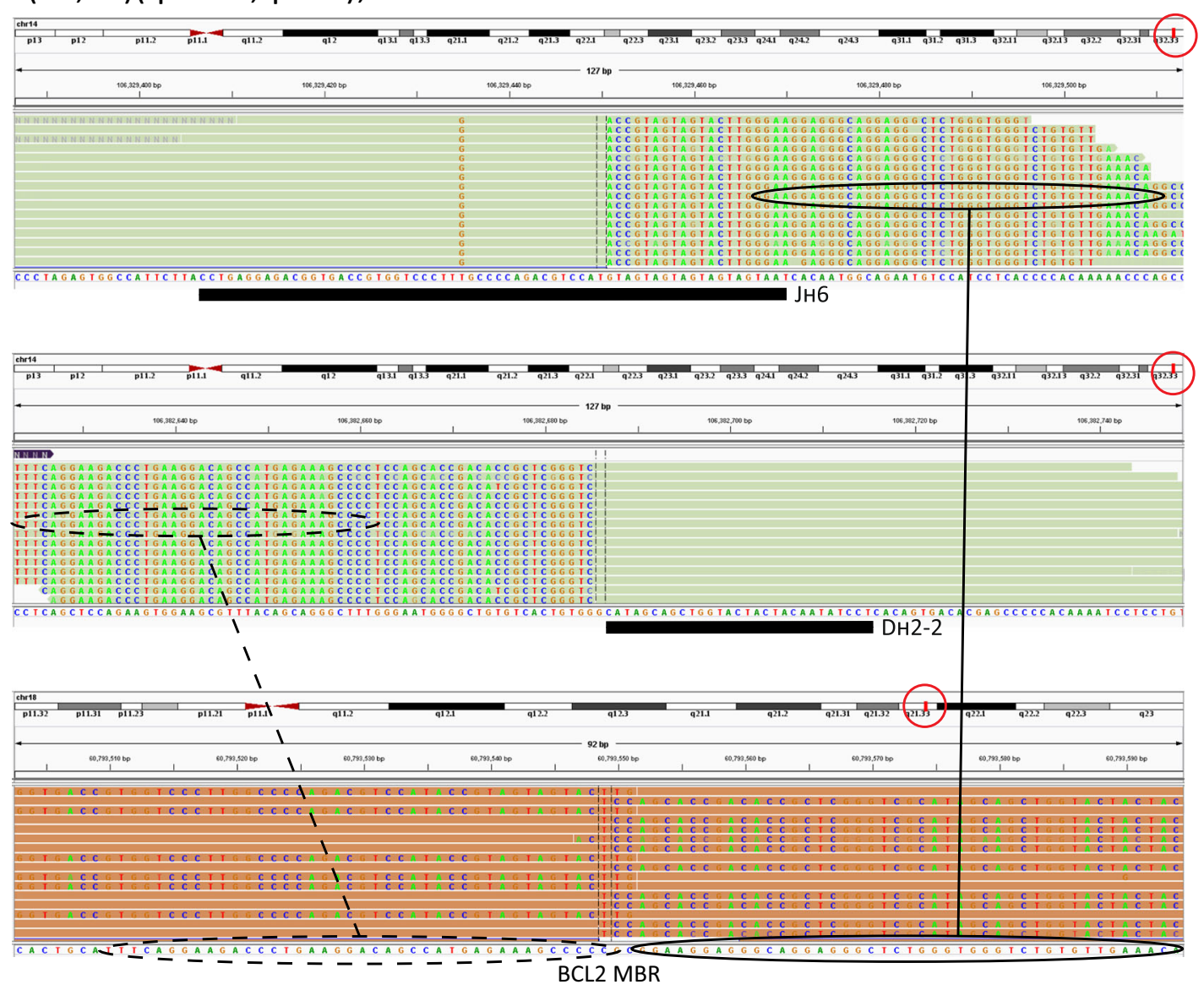


Fig. 2 Alignment of split reads. Each panel from the IGV program shows sequencing reads aligned to a particular chromosomal location. The reads were "colored by insert size" and "grouped by chromosome of mate." The structure of the entire chromosome is shown at the top of each panel with the location of the gene circled in red. The reference sequence is shown at the bottom of each panel. Genes or regions in the area of alignment are indicated below each panel. The color of the reads indicates to which chromosome the discordant read pair maps. Bases listed in the reads indicate mismatches. Circled sequences indicate how the mismatched portion from split reads aligns to the reference of the other chromosome. Solid and dashed circles and lines indicate sequences from the two different derivative chromosomes. a $t(3 ; 16)$; BCL6-CIITA. This fusion occurs between a $\mathrm{p}$ and a $\mathrm{q}$ arm; therefore, the circled sequences are the reverse complement of each other. $\mathbf{b} t(14 ; 18)$; $I G H-B C L 2$. In this typical fusion, two breaks occurred on chromosome 14 with deletion of the intervening portion. Sequences from the two derivative chromosomes (solid and dashed circles and lines) involving $B C L 2 \mathrm{MBR}$ align to separate regions on chromosome 14

Routine microdissection, especially for extranodal cases, may increase the sensitivity of this test. It is also possible that for some rearrangements, especially those involving $M Y C$ and a non-Ig partner, both breakpoints may not be captured by our current design. Several $M Y C$ breakpoints have been identified far upstream and downstream of the gene, outside the well-defined major translocation cluster (MTC), including those involving light chain fusions [24-27]. This should not pose a problem when an Ig locus with its well-defined breakpoints is involved (e.g., cases 15, 53), but a proportion of $M Y C$ translocations involve a non-IG partner [28], for example case 22, and if some of these events involve noncaptured breakpoints mapping outside the MTC, they would not be detected by sequencing. In this case, the advantage of break-apart FISH analysis lies in the ability to capture $M Y C$ breakpoints dispersed over megabase distances even when involved with an unknown fusion partner. A large number of MYC break-apart FISH-positive cases will need to be sequenced in order to determine the fraction of these events that are not detectable by sequencing. A recent study involving sequencing of several megabases around the $M Y C$ locus further elucidates the wide distribution of breakpoints [29]. Very rare breakpoints have also been described downstream of $C_{H} \mu$ outside the captured class switch regions [24] which could also be a possible reason for failure. Lastly, several of the captured regions belong to gene families (for example V segments) or are composed of low-complexity
Fig. 3 Fusion gene structures. a Schematic (not to scale) of $B C L 2$ and portion of the $I G H$ locus on chromosomes 18 and 14, respectively. The der(14) harboring the putative inverted insertion of BCL2 in case 31 is shown. The approximate locations of hybridization of the green and red FISH probes are shown above the genes (dashed red and green lines, IGH-BCL2 fusion probes; solid red and green lines, $I G H$ break-apart probes). $\mathbf{b}$ Schematic structure of the $5^{\prime}$ portion of $B C L 6$ (in black) and its fusions with CIITA, NACA, and DLEU2. Thick lines indicate the primary transcripts with boxes depicting the exons. Coding exons are shown larger. The structures of the final spliced products and translated proteins have not been determined a
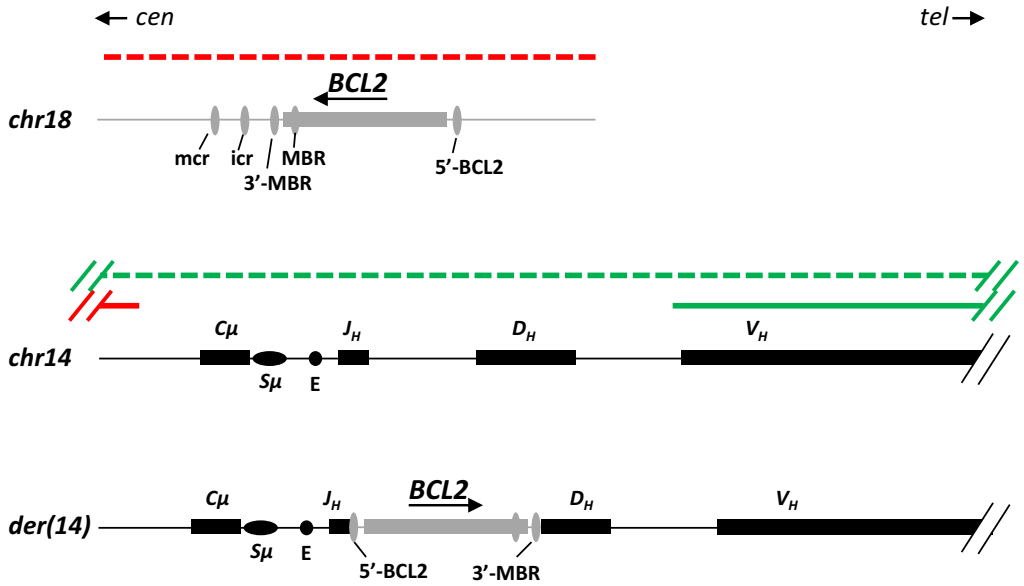

b
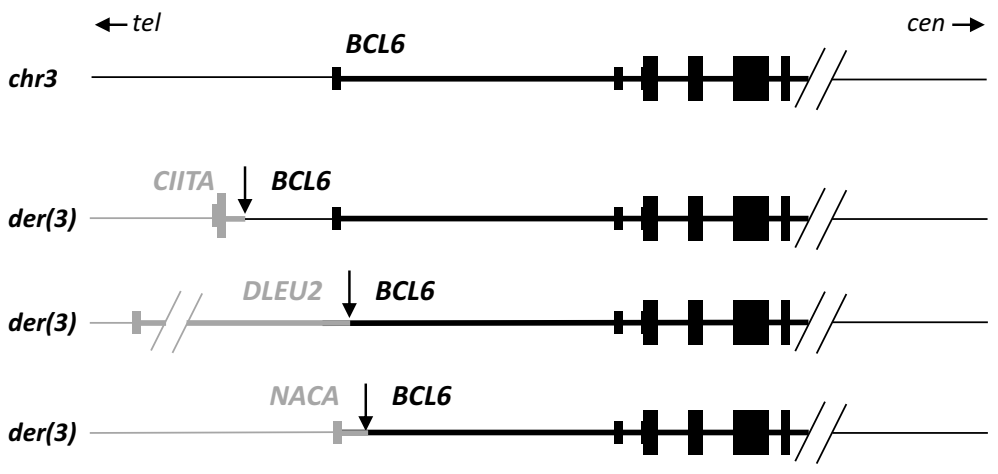
Fig. 4 Distribution of breakpoints in the BCL2 Locus. Schematic (not to scale) of the BCL2 locus with the 5 known breakpoint cluster regions. Triangles indicate the location of each detected breakpoint. Open triangles map outside the captured regions. Thick lines indicate the primary transcripts with boxes depicting the exons. Coding exons are shown larger. Mcr, minor cluster region; icr, intermediate cluster region; MBR, major breakpoint region

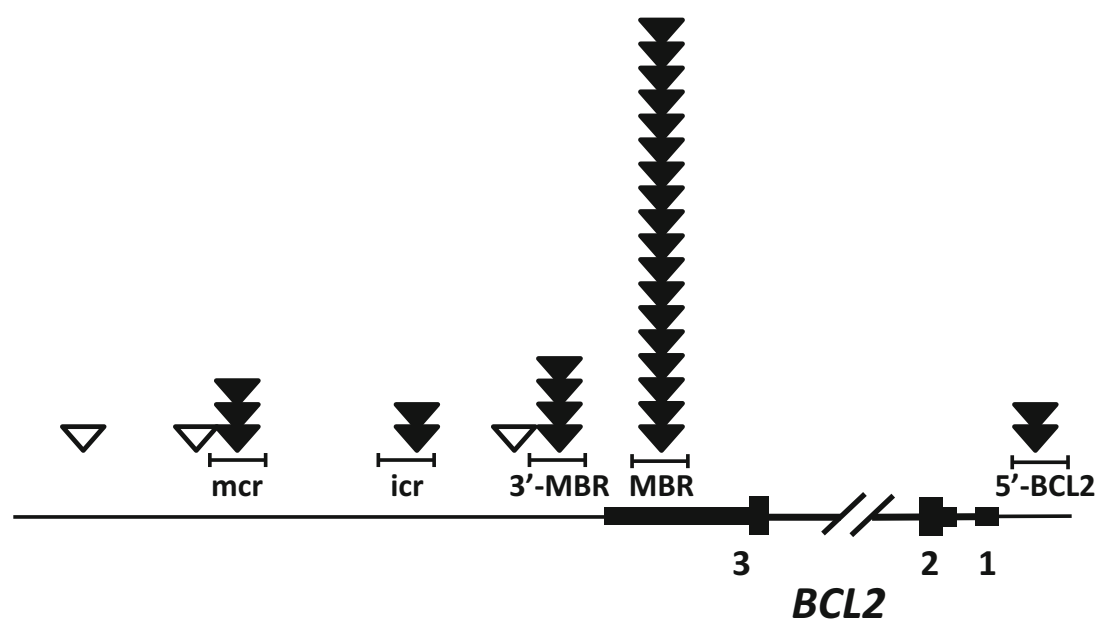

sequence (for example, $I G H$ class switch regions). Sequencing reads from these regions have reduced mapping quality, resulting in a loss of sensitivity, possibly explaining the failure to detect the $I G H$ breakpoint in case 46. Despite the potential for poor mapping quality of reads to some of these regions, we could detect a total of 17 translocations involving 6 out of the 8 class switch regions.

A recent report indicates the possibility that the identity of the fusion partner in $M Y C$ translocation may determine the prognostic impact of the rearrangement, especially in the scenario of double-hit translocations [28]. Sequencing has the advantage of identifying the fusion partner in such scenarios. Detection of translocations by sequencing may allow further stratification of rearrangements currently grouped as a single entity by break-apart FISH analysis.

An alternative approach to solution capture used for target enrichment in this study is anchored multiplex PCR (ArcherDX, Inc., Boulder, CO) which uses only a single gene-specific PCR primer and a universal primer to a ligated adapter. This variation of PCR does allow for the enrichment of unknown fusion partners as opposed to standard ampliconbased methods [30, 31]. The method works best for RNA sequencing applications due to the short targets in spliced transcripts, including the non-Ig BCL6 fusions described here, 3'-UTR fusions of BCL2 when the major breakpoint region (MBR) is involved, and breakpoints in the BCL6 and MYC MTCs that map within the respective 5'-UTRs. The method may be difficult to adapt to larger genomic areas, such as the $I G H$ switch regions or other areas at a distance from transcribed sequences. RNA expression analysis by RNA sequencing may be a surrogate marker for the presence of enhancer fusions to proto-oncogenes, such as IGH-MYC.

Overall, we believe that FISH analysis and massively parallel sequencing are mostly complementary and possibly should be used in conjunction. As evidence of this, sequencing aids in the clarification of atypical FISH patterns, as shown here for 3 cases. Many additional cases will need to be tested in order to determine any recurrent correlations between atypical FISH patterns and sequencing results.

Funding This study was supported by the Association for Regional and University Pathologists (ARUP) Laboratories, Salt Lake City, UT.

\section{Compliance with ethical standards}

Conflict of interest The authors declare that they have no conflict of interest.

\section{References}

1. Kuppers R, Dalla-Favera R (2001) Mechanisms of chromosomal translocations in B cell lymphomas. Oncogene 20(40):5580-5594. https://doi.org/10.1038/sj.onc. 1204640

2. Goossens T, Klein U, Kuppers R (1998) Frequent occurrence of deletions and duplications during somatic hypermutation: implications for oncogene translocations and heavy chain disease. Proc Natl Acad Sci U S A 95(5):2463-2468

3. Espinet B, Bellosillo B, Melero C, Vela MC, Pedro C, Salido M, Pijuan L, Florensa L, Besses C, Serrano S, Sole F (2008) FISH is better than BIOMED-2 PCR to detect IgH/BCL2 translocation in follicular lymphoma at diagnosis using paraffin-embedded tissue sections. Leuk Res 32(5):737-742. https://doi.org/10.1016/j. leukres.2007.09.010

4. Shen W, Szankasi P, Sederberg M, Schumacher J, Frizzell KA, Gee EP, Patel JL, South ST, Xu X, Kelley TW (2016) Concurrent detection of targeted copy number variants and mutations using a myeloid malignancy next generation sequencing panel allows comprehensive genetic analysis using a single testing strategy. Br J Haematol 173(1):49-58. https://doi.org/10.1111/bjh.13921

5. Akasaka H, Akasaka T, Kurata M, Ueda C, Shimizu A, Uchiyama T, Ohno H (2000) Molecular anatomy of BCL6 translocations revealed by long-distance polymerase chain reaction-based assays. Cancer Res 60(9):2335-2341

6. Ohno H (2006) Pathogenetic and clinical implications of non-immunoglobulin: BCL6 translocations in B-cell non-Hodgkin's lymphoma. J Clin Exp Hematopathol : JCEH 46(2):43-53

7. Bouamar H, Abbas S, Lin AP, Wang L, Jiang D, Holder KN, Kinney MC, Hunicke-Smith S, Aguiar RC (2013) A capture- 
sequencing strategy identifies IRF8, EBF1, and APRIL as novel IGH fusion partners in B-cell lymphoma. Blood 122(5):726-733. https://doi.org/10.1182/blood-2013-04-495804

8. Akasaka T, Akasaka H, Yonetani N, Ohno H, Yamabe H, Fukuhara S, Okuma M (1998) Refinement of the BCL2/immunoglobulin heavy chain fusion gene in $\mathrm{t}(14 ; 18)(\mathrm{q} 32 ; \mathrm{q} 21)$ by polymerase chain reaction amplification for long targets. Genes Chromosom Cancer 21(1):17-29

9. Burmeister T, Molkentin M, Schwartz S, Gokbuget N, Hoelzer D, Thiel E, Reinhardt R (2013) Erroneous class switching and false VDJ recombination: molecular dissection of $\mathrm{t}(8 ; 14) / \mathrm{MYC}-\mathrm{IGH}$ translocations in Burkitt-type lymphoblastic leukemia/B-cell lymphoma. Mol Oncol 7(4):850-858. https://doi.org/10.1016/j. molonc.2013.04.006

10. Albinger-Hegyi A, Hochreutener B, Abdou MT, Hegyi I, DoursZimmermann MT, Kurrer MO, Heitz PU, Zimmermann DR (2002) High frequency of $\mathrm{t}(14 ; 18)$-translocation breakpoints outside of major breakpoint and minor cluster regions in follicular lymphomas: improved polymerase chain reaction protocols for their detection. Am J Pathol 160(3):823-832. https://doi.org/10.1016/S00029440(10)64905-X

11. Murga Penas EM, Callet-Bauchu E, Ye H, Gazzo S, Berger F, Schilling G, Albert-Konetzny N, Vettorazzi E, Salles G, Wlodarska I, Du MQ, Bokemeyer C, Dierlamm J (2010) The $\mathrm{t}(14 ; 18)(\mathrm{q} 32 ; \mathrm{q} 21) /$ IGH-MALT1 translocation in MALT lymphomas contains templated nucleotide insertions and a major breakpoint region similar to follicular and mantle cell lymphoma. Blood 115(11):2214-2219. https://doi.org/10.1182/blood-2009-08236265

12. Fan H, Gulley ML, Gascoyne RD, Horsman DE, Adomat SA, Cho CG (1998) Molecular methods for detecting $t(11 ; 14)$ translocations in mantle-cell lymphomas. Diagn Mol Pathol 7(4):209-214

13. Baens M, Steyls A, Dierlamm J, De Wolf-Peeters C, Marynen P (2000) Structure of the MLT gene and molecular characterization of the genomic breakpoint junctions in the $\mathrm{t}(11 ; 18)(\mathrm{q} 21 ; \mathrm{q} 21)$ of marginal zone B-cell lymphomas of MALT type. Genes Chromosom Cancer 29(4):281-291

14. Yonetani N, Ueda C, Akasaka T, Nishikori M, Uchiyama T, Ohno $H$ (2001) Heterogeneous breakpoints on the immunoglobulin genes are involved in fusion with the $5^{\prime}$ region of BCL2 in B-cell tumors. Japn J Cancer Res : Gann 92(9):933-940

15. Li H (2013) Aligning sequence reads, clone sequences and assembly contigs with BWA-MEM. arXiv

16. Rausch T, Zichner T, Schlattl A, Stutz AM, Benes V, Korbel JO (2012) DELLY: structural variant discovery by integrated pairedend and split-read analysis. Bioinformatics 28(18):i333-i339. https://doi.org/10.1093/bioinformatics/bts378

17. Liew M, Rowe L, Clement PW, Miles RR, Salama ME (2016) Validation of break-apart and fusion MYC probes using a digital fluorescence in situ hybridization capture and imaging system. J Pathol Inform 7:20. https://doi.org/10.4103/2153-3539.181764

18. Ueda C, Akasaka T, Kurata M, Maesako Y, Nishikori M, Ichinohasama R, Imada K, Uchiyama T, Ohno H (2002) The gene for interleukin-21 receptor is the partner of BCL6 in $\mathrm{t}(3 ; 16)(\mathrm{q} 27 ; \mathrm{p} 11)$, which is recurrently observed in diffuse large B-cell lymphoma. Oncogene 21(3):368-376. https://doi.org/10. 1038/sj.onc.1205099

19. Yoshida S, Kaneita Y, Aoki Y, Seto M, Mori S, Moriyama M (1999) Identification of heterologous translocation partner genes fused to the BCL6 gene in diffuse large B-cell lymphomas: 5'-RACE and LA - PCR analyses of biopsy samples. Oncogene 18(56):7994 7999. https://doi.org/10.1038/sj.onc.1203293

20. Bakhshi A, Wright JJ, Graninger W, Seto M, Owens J, Cossman J, Jensen JP, Goldman P, Korsmeyer SJ (1987) Mechanism of the $\mathrm{t}(14 ; 18)$ chromosomal translocation: structural analysis of both derivative 14 and 18 reciprocal partners. Proc Natl Acad Sci U S A 84(8):2396-2400

21. Halper-Stromberg E, Steranka J, Giraldo-Castillo N, Fuller T, Desiderio S, Burns KH (2013) Fine mapping of V(D)J recombinase mediated rearrangements in human lymphoid malignancies. BMC Genomics 14:565. https://doi.org/10.1186/1471-2164-14-565

22. Cotter F, Price C, Zucca E, Young BD (1990) Direct sequence analysis of the 14q+ and 18q- chromosome junctions in follicular lymphoma. Blood 76(1):131-135

23. Vaandrager JW, Schuuring E, Philippo K, Kluin PM (2000) V(D)J recombinase-mediated transposition of the BCL2 gene to the IGH locus in follicular lymphoma. Blood 96(5):1947-1952

24. Joos S, Falk MH, Lichter P, Haluska FG, Henglein B, Lenoir GM, Bornkamm GW (1992) Variable breakpoints in Burkitt lymphoma cells with chromosomal $\mathrm{t}(8 ; 14)$ translocation separate c-myc and the IgH locus up to several hundred kb. Hum Mol Genet 1(8): 625-632

25. Joos S, Haluska FG, Falk MH, Henglein B, Hameister H, Croce CM, Bornkamm GW (1992) Mapping chromosomal breakpoints of Burkitt's $\mathrm{t}(8 ; 14)$ translocations far upstream of c-myc. Cancer Res 52(23):6547-6552

26. Einerson RR, Law ME, Blair HE, Kurtin PJ, McClure RF, Ketterling RP, Flynn HC, Dogan A, Remstein ED (2006) Novel FISH probes designed to detect IGK-MYC and IGL-MYC rearrangements in B-cell lineage malignancy identify a new breakpoint cluster region designated BVR2. Leukemia 20(10):1790-1799. https://doi.org/10.1038/sj.leu.2404340

27. Henglein B, Synovzik H, Groitl P, Bornkamm GW, Hartl P, Lipp M (1989) Three breakpoints of variant $\mathrm{t}(2 ; 8)$ translocations in Burkitt's lymphoma cells fall within a region 140 kilobases distal from cmyc. Mol Cell Biol 9(5):2105-2113

28. Copie-Bergman C, Cuilliere-Dartigues P, Baia M, Briere J, Delarue R, Canioni D, Salles G, Parrens M, Belhadj K, Fabiani B, Recher C, Petrella T, Ketterer N, Peyrade F, Haioun C, Nagel I, Siebert R, Jardin F, Leroy K, Jais JP, Tilly H, Molina TJ, Gaulard P (2015) MYC-IG rearrangements are negative predictors of survival in DLBCL patients treated with immunochemotherapy: a GELA/ LYSA study. Blood 126(22):2466-2474. https://doi.org/10.1182/ blood-2015-05-647602

29. Chong LC, Ben-Neriah S, Slack GW, Freeman C, Ennishi D, Mottok A, Collinge B, Abrisqueta P, Farinha P, Boyle M, Meissner B, Kridel R, Gerrie AS, Villa D, Savage KJ, Sehn LH, Siebert R, Morin RD, Gascoyne RD, Marra MA, Connors JM, Mungall AJ, Steidl C, Scott DW (2018) High-resolution architecture and partner genes of MYC rearrangements in lymphoma with DLBCL morphology. Blood Adv 2(20):2755-2765. https://doi.org/ 10.1182/bloodadvances.2018023572

30. Zhu G, Benayed R, Ho C, Mullaney K, Sukhadia P, Rios K, Berry R, Rubin BP, Nafa K, Wang L, Klimstra DS, Ladanyi M, Hameed MR (2019) Diagnosis of known sarcoma fusions and novel fusion partners by targeted RNA sequencing with identification of a recurrent ACTB-FOSB fusion in pseudomyogenic hemangioendothelioma. Mod Pathol 32(5):609-620. https://doi.org/10.1038/s41379-0180175-7

31. Kim B, Lee H, Shin S, Lee ST, Choi JR (2019) Clinical evaluation of massively parallel RNA sequencing for detecting recurrent gene fusions in hematologic malignancies. J Mol Diagn 21(1):163-170. https://doi.org/10.1016/j.jmoldx.2018.09.002

Publisher's note Springer Nature remains neutral with regard to jurisdictional claims in published maps and institutional affiliations. 